\title{
Terapia miofuncional en quemaduras orofaciales de segundo y tercer grado
}

\author{
Myofunctional therapy for 2nd and 3rd degree \\ orofacial burns
}

\begin{abstract}
Axel Pavez
Hospital de Urgencia Asistencia

Pública
\end{abstract}

\section{Contacto con el autor: Axel Pavez Santiago - Chile Correo-e: axel.pavez@redsalud.gob.cl}

Recibido: 06/08/2018 Aceptado: 17/10/2018

\section{RESUMEN}

Las quemaduras constituyen lesiones traumáticas que comprometen la piel. Aquellas que involucran la región facial son las más complejas, dada la relevancia estética y funcional de esta zona, afectando la expresión de emociones, así como procesos fundamentales como la visión, habla, alimentación, entre otros. Pese a la escasa evidencia, se ha demostrado que la terapia fonoaudiológica miofuncional disminuye las secuelas en pacientes con quemaduras orofaciales. En este contexto, la presente investigación tiene como objetivo describir y evidenciar los efectos de la terapia fonoaudiológica miofuncional en quemaduras orofaciales de $2^{\circ}$ y $3 e r$ grado. Para ello, se incluyeron 10 sujetos con quemaduras orofaciales de $2^{\circ}$ y $3 e r$ grado ingresados al Centro de Referencia Nacional de Quemados en Santiago de Chile, quienes recibieron evaluación y terapia miofuncional por un fonoaudiólogo durante su hospitalización. El 80\% de los sujetos presentaban quemaduras de $2^{\circ}$ grado y $20 \%$ de $3^{\circ}$ grado, el $30 \%$ requirió injerto facial. En la evaluación inicial se observó disminución de la apertura bucal vertical y horizontal, además de disminución en los movimientos de la mímica facial en la totalidad de los casos. Luego de la intervención se evidenciaron mejorías en todos los parámetros, incluido además el nivel de autopercepción de retracción pre y post terapia fonoaudiológica; todos con diferencias estadísticamente significativas $(\mathrm{p}<0,05)$.

Estos resultados nos permiten concluir que la terapia fonoaudiológica miofuncional favorece parámetros como apertura bucal y movilidad facial en pacientes con quemaduras orofaciales. La inclusión de fonoaudiólogos capacitados en el abordaje de estas personas, sería beneficioso para aminorar secuelas y beneficiar su calidad de vida.

Palabras clave: quemaduras, terapia miofuncional, sistema estomatognático, fonoaudiología, rehabilitación.

\begin{abstract}
Burns are traumatic injuries that compromise skin. Facial burns are the most complex burns, because of this area's aesthetic and functional relevance. This kind of injuries affect emotion expression and relevant processes such as vision, speech, and feeding, among others. Despite scarce evidence, it has been shown that myofunctional speech-language therapy decreases sequelae among patients with orofacial burns. This study, therefore, set out to describe the myofunctional speech-language therapy intervention in 2nd and 3rd degree facial burns. 10 patients with 2nd and 3rd degree facial burns admitted to National Burn Center in Santiago, Chile, were recruited for this study. $80 \%$ of subjects had 2 nd degree burns and $20 \% 3$ rd degree burns. $30 \%$ of subjects required a facial skin graft. At initial assessment, there was a decrease in the vertical and horizontal mouth opening, as well as a decrease in facial movements in all cases. All the subjects received a myofunctional speech-language therapy intervention during the hospitalization period. On the completion of myofunctional intervention, patients improved in all parameters, with statistically significant differences $(p<0,05)$, in addition to self-perception level of retraction pre and post therapy. The results of this study show that myofunctional speechlanguage therapy improves the performance in parameters such as mouth opening and facial movements among patients with orofacial burns. The inclusion of trained Speech-language therapists is helpful for interventions focused on persons with burns, inasmuch as decreases sequelae and improves quality of life.
\end{abstract}

Keywords: burns, myofunctional therapy, stomatognathic system, speech-language pathology, rehabilitation. 


\section{Introducción}

La región facial presenta gran relevancia para el ser humano, pues establece el punto central de las características físicas y contribuye en la identidad individual. Además, participa en la comunicación humana por medio de la expresión de emociones y en otros procesos fundamentales como visión, habla, alimentación, entre otros.

Por su parte una quemadura constituye una lesión traumática que involucra la destrucción total o parcial de alguna de las capas de la piel; pudiendo ocasionar la muerte del individuo o secuelas a nivel funcional, estético, emocional y social.

Las quemaduras se pueden clasificar de acuerdo al agente etiológico que las produce, la profundidad (nivel de compromiso histológico), extensión (superficie corporal quemada o SCQ) y las zonas corporales involucradas.

De acuerdo a lo establecido en la guía clínica del paciente quemado de la International Society for Burn Injury (ISBI Practice Guidelines Committee, Steering Subcommittee, \& Advisory Subcommittee, 2016), las quemaduras faciales constituyen quemaduras en zonas especiales que requieren manejo con equipo especializado, debido a la relevancia funcional y estética de esta zona. Sumado a lo anterior, el riesgo inminente de injuria inhalatoria en aquellos pacientes con quemadura facial, aumenta la complejidad, gravedad y empeora el pronóstico vital.

Las quemaduras faciales alteran las estructuras anatómicas y funcionales ocasionando dolor, deformidad y/o edema. En casos severos y dependiendo de la profundidad y extensión, es posible evidenciar retracciones o contracturas que pueden conducir secuelas físicas, estéticas y psicológicas permanentes (Hop et al., 2014; Stoddard, Ryan, \& Schneider, 2014). El tratamiento debe ser siempre realizado por un equipo especializado e interdisciplinario, quirúrgico o no quirúrgico, y debe incluir profesionales de diversas áreas que trabajen en conjunto con el objetivo de salvar la vida de la persona, mejorar los resultados funcionales $y$ estéticos, disminuir el riesgo de secuelas o tratar las mismas en forma precoz y adecuada (Wei \& Li-Tsang, 2017).

Los aportes de la fonoaudiología en el trabajo con quemados inicia cercano a la década del 90 (Brooks \& Hammond, 1986; Williams \& Baker, 1992). No obstante, su participación activa, según lo descrito en la literatura, no supera los 20 años. La contribución del fonoaudiólogo en la intervención del paciente quemado se enmarca en las áreas de deglución, comunicación (habla y voz) y motricidad orofacial (MOF) (Rumbach, Clayton, Muller, \& Maitz, 2016). En esta última área, el trabajo se enfoca en el manejo y prevención de retracciones y cicatrices hipertróficas en la región orofacial (Rumbach et al., 2016; Snyder \& Ubben, 2003), además de favorecer la movilidad y funcionalidad de la zona.

La evidencia a nivel internacional aún es limitada, con pocos artículos científicos referidos al área de motricidad orofacial pero que muestran una significativa contribución de la profesión en este grupo de pacientes (Borges, Vieira, \& Barreto, 2011; Clayton, Ellul, Ward, Scott, \& Maitz, 2017; Clayton, Ward, \& Maitz, 2015a, 2015b; Magnani, Sassi, Vana, Alonso, \& Andrade, 2015; Ramos, Danda, Araujo, Regis, \& Silva, 2009; Toledo, 2003).

Las quemaduras orofaciales de $2^{\circ}$ y $3^{\text {er }}$ grado han 
demostrado presentar mayor déficit en relación a las estructuras orofaciales, rango de movimientos mandibulares y de la mímica facial (Magnani et al., 2015), pudiendo interferir en procesos de comunicación (verbal y no verbal) y/o alimentación. La disminución de los movimientos de la mímica facial cobra relevancia desde el punto de vista comunicativo, ya que la comunicación no verbal contribuye a una mejora en la comprensión del mensaje por medio del uso de gestos y expresiones faciales. Por su parte, el proceso de cicatrización patológica puede producir contracturas secundarias, las cuales pueden comprometer el rango de los movimientos horizontales y/o verticales de apertura bucal, ocasionando incluso microstomía en casos severos (Clayton et al., 2015a, 2015b). Una completa funcionalidad de la apertura bucal considera la posibilidad de articular en forma adecuada, comer sin restricciones, beber líquidos sin escape anterior $y$ cepillarse los dientes con acceso total a los molares. Para ello se considera como valores de referencia de funcionalidad de la apertura bucal, $40 \mathrm{~mm}$ vertical y $50 \mathrm{~mm}$ horizontal (Clayton et al., 2015a; Mezitis, Rallis, \& Zachariades, 1989).

En Chile y gran parte del mundo, a excepción de Australia, Estados Unidos y Brasil (países con mayor contribución científica en el área), este tema constituye un área de intervención emergente y desconocida, tanto para los mismos fonoaudiólogos como para el resto de los profesionales que trabajan con quemados. A la fecha, en nuestro país sólo existe un reporte de caso (Pavez \& Tobar, 2016) que relata de manera sucinta la intervención fonoaudiológica realizada en un paciente gran quemado de $53 \%$ de superficie quemada y con compromiso orofacial, que presentó alteraciones a nivel deglutorio, fonatorio y de motricidad orofacial. Pese a ello, la información disponible a nivel nacional y referida a la atención fonoaudiológica propiamente tal, sigue siendo escasa.

El Servicio de Quemados del Hospital de Urgencia Asistencia Pública (HUAP) constituye el centro de referencia nacional del paciente gran quemado adulto, recibiendo cerca del $90 \%$ de los pacientes quemados graves de todo el país. Es el único centro para pacientes adultos beneficiarios del sistema público de salud, entregando atención a todos quienes cumplen con los criterios de gran quemado según lo establecido en las guías clínicas del programa de garantías explícitas en salud (GES) (Albornoz, Villegas, Peña, \& Whittle, 2013; Ministerio de Salud de Chile, 2016). Estas últimas, no consideran al fonoaudiólogo como parte del equipo de salud en la atención del paciente quemado (Ministerio de Salud de Chile, 2016).

En vista de lo descrito y con la intención de contribuir en el conocimiento con que cuentan los fonoaudiólogos, además de potenciar el desarrollo de esta área y proveer evidencia en relación a esta temática; el presente artículo tiene por objetivo describir y evidenciar los efectos de la terapia fonoaudiológica miofuncional en un grupo de personas con quemaduras orofaciales de $2^{\circ}$ y $3^{\text {er }}$ grado.

\section{Metodología}

\section{Participantes}

Se desarrolló un estudio descriptivo correspondiente a una serie de 10 casos (ver tabla 1 ), donde se analizó en forma retrospectiva la base de 
datos de Fonoaudiología de pacientes atendidos en el servicio de quemados del Hospital de Urgencia Asistencia Pública (HUAP), Centro de Referencia Nacional de Quemados en Santiago de Chile en un periodo de 10 meses (Marzo a Diciembre de 2016).

\section{Criterios de inclusión y exclusión}

Se incluyeron todos aquellos pacientes con quemadura orofacial de $2^{\circ}$ y $3^{\text {er }}$ grado con diagnóstico de desorden miofuncional orofacial y que recibieron intervención fonoaudiológica miofuncional durante el periodo seleccionado.

Se excluyeron aquellos pacientes que hubieran recibido apoyo con expansores bucales.

\section{Procedimientos e instrumentos}

Si bien, considerando que de acuerdo al nivel de compromiso de los casos incorporados en el estudio, todos recibieron intervención fonoaudiológica integral (lo cual implica un abordaje de deglución, voz y/o motricidad orofacial; según resultados en la evaluación inicial), para fines del artículo sólo se describirá la intervención a nivel de motricidad orofacial.

La evaluación fonoaudiológica inicial fue realizada en fase hospitalaria, una vez que los pacientes se encontraron médicamente estables. Esta evaluación considera un screening en los distintos ámbitos, entregando una panorámica global sobre las áreas a abordar.

Respecto a la evaluación miofuncional, esta consideró aspectos clínicos generales de la motricidad orofacial, movimientos de mímica facial y medidas antropométricas. Fue utilizada una pauta clínica fonoaudiológica de pacientes quemados diseñada por el autor. Esta pauta incluye exploración extraoral (simetría facial, indemnidad de órganos fonoarticulatorios, sensibilidad, retracciones), exploración intraoral (dentición y oclusión, presencia de contractura, dolor a la palpación) y funciones estomatognáticas (respiración, succión, masticación y articulación). De acuerdo a la condición clínica del paciente algunos procedimientos de evaluación debieron ser adaptados, o bien dividir la evaluación en más de una oportunidad; limitando la igualdad en las condiciones de evaluación. En este sentido, y sumado a lo subjetivo y cualitativo de este ítem, se optó por no considerar estos hallazgos para el análisis.

Todos los pacientes recibieron evaluación de medidas antropométricas (apertura bucal vertical y horizontal) y mímica facial, de acuerdo a lo propuesto por Toledo (2003). Las medidas antropométricas fueron realizadas con un paquímetro o pie de metro, mientras que para la evaluación de la mímica facial fue utilizado el Clinical Score for Facial Mimic Protocol (Salles, Toledo, \& Ferreira, 2009), que considera una serie de movimientos voluntarios e involuntarios a nivel facial con un puntaje total de 40 puntos (ver figura 1). 

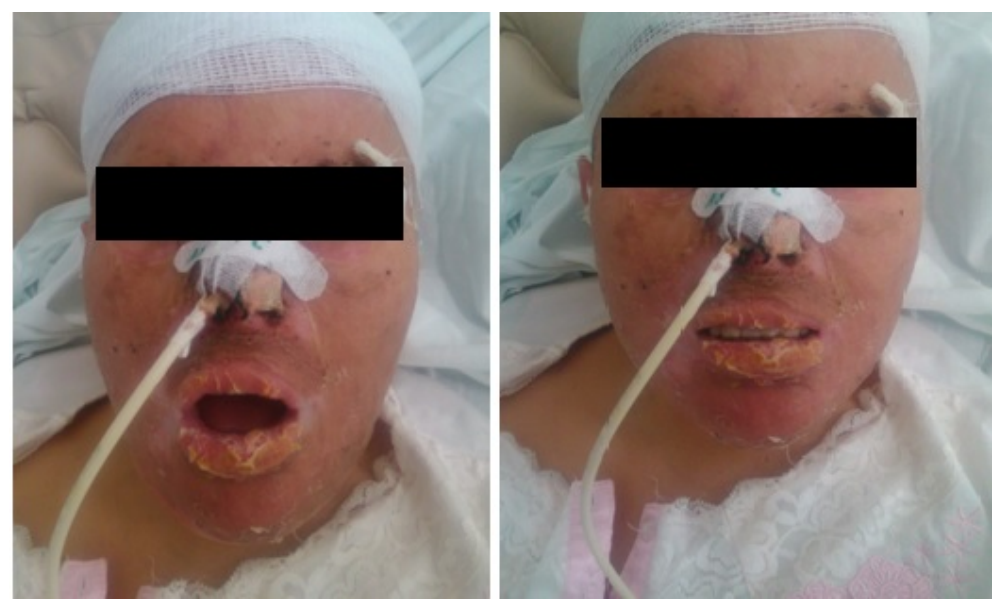

Figura 1. Paciente con quemadura orofacial de 3er grado (profundo) por fuego, con injerto en zona facial, realizando movimientos orofaciales de apertura bucal (fig. izq.) y sonrisa (fig. der.); de acuerdo con el protocolo de Salles et al. (2009).

Fig. Izquierda: Se observa disminución de apertura bucal y cicatriz en ambas comisuras. Fig. Derecha: Se observa disminución del rango del movimiento (R.O.M) horizontal y mímica facial. (Imagen autorizada por paciente)

La terapia miofuncional tuvo como objetivo evitar la retracción del tejido y los patrones compensatorios negativos a nivel orofacial que pueden interferir en la comunicación y deglución, además de promover el equilibrio de las funciones dependientes del sistema estomatognático. El tratamiento se consideró finalizado cuando el valor en las medidas antropométricas se encontró cercano y/o dentro de los rangos esperables y no existía compromiso en la funcionalidad a nivel de comunicación o alimentación.
En aquellos casos donde aún no se completaban los objetivos propuestos, el tratamiento se consideró inconcluso.

Para el cumplimiento de los objetivos se utilizaron ejercicios orofaciales activos, activos con asistencia, técnicas de manipulación digital y masoterapia (ver figura 2). Para asegurar la efectividad de la intervención miofuncional, no se utilizaron expansores bucales que pudieran interferir en las variables en estudio.
A

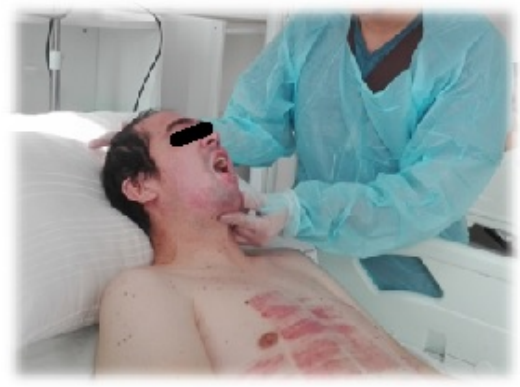

B

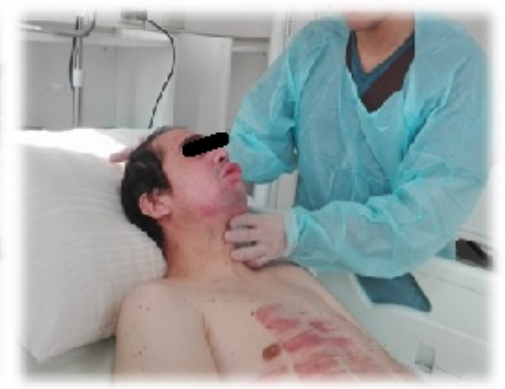

C

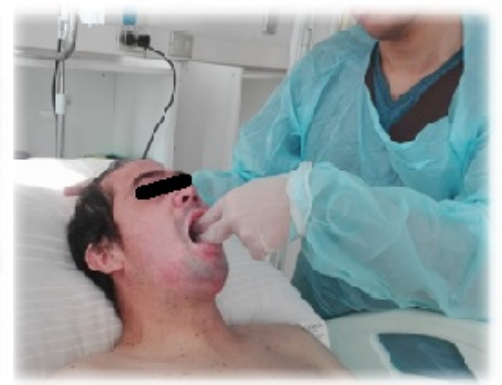

Figura 2. (A) y (B) Ejercicios orofaciales activos. (C) Ejercicios orofaciales activos con asistencia en paciente con quemadura orofacial de grado intermedio reepidermizada. (Imágenes autorizadas por paciente) 
Posterior a la evaluación y el diagnóstico se consideraron 1 a 2 sesiones semanales de terapia directa (dependiendo de la disponibilidad clínica u otros procedimientos del equipo), con una duración de entre 20 a 25 minutos. Además de terapia indirecta que consistía en la replicación de algunos ejercicios trabajados en la sesión fonoaudiológica con un programa de ejercicios entregados a cada usuario.

En cada sesión el paciente determinaba de manera subjetiva el nivel de autopercepción de retracción, mediante la entrega de respuestas cerradas del tipo sí/no. Además, se solicitaba la valoración del nivel de retracción con escala de 1 a 7 (donde 1 constituía sensación de máxima retracción y 7 sensación de mínima retracción). Esta información era solicitada al inicio y término de la sesión fonoaudiológica.

Toda la información fue almacenada en la base de datos de Fonoaudiología, la cual sólo incluye información relevante al contexto clínico hospitalario y excluye datos sensibles; según lo propuesto por la Ley 20.584 de deberes y derechos de los pacientes en conformidad a la Ley 19.628 sobre la protección de la vida privada. Así mismo, no se requirió revisión de ficha ni acceso a información sensible por otros medios.

\section{Análisis estadístico}

El análisis estadístico se realizó utilizando estadística descriptiva y prueba de Wilcoxon para comparación de variables cuantitativas (valor $p<0,05$ ), a través del software estadístico SPSS ${ }^{\circledR}$ V. 22.0.

\section{Resultados}

A continuación, se describen las características clínicas y condiciones sociodemográficas de los 10 casos considerados en el estudio (tabla 1 ). 
Tabla 1

Caracterización de los casos en relación condiciones sociodemográficas, datos de la quemadura y datos de la intervención médica/fonoaudiológica $(n=10)$.

\begin{tabular}{|c|c|c|c|c|c|c|c|c|c|c|}
\hline Caso & Género & $\begin{array}{l}\text { Edad } \\
\text { (años) }\end{array}$ & $\begin{array}{c}\text { Extensión } \\
\text { (\% SCQ) }\end{array}$ & $\begin{array}{l}\text { Profundidad } \\
\text { (Grado) }\end{array}$ & Mecanismo & I.I & IOT/TQT & IDE & $\begin{array}{c}\mathrm{N}^{\circ} \\
\mathrm{MOF}\end{array}$ & $\begin{array}{l}\text { Procedencia } \\
\text { (Región) }\end{array}$ \\
\hline 1 & M & 55 & $9 \%$ & $2^{\circ}$ & Mixto $(F+E)$ & Sí & Ambas & Sí & 6 & Ovalle (IV) \\
\hline 2 & M & 49 & $36 \%$ & $2^{\circ}$ & Explosión & Sí & IOT & No & 4 & $\begin{array}{c}\text { Independencia } \\
\text { (RM) }\end{array}$ \\
\hline 3 & M & 28 & $51 \%$ & $2^{\circ}$ & Explosión & Sí & IOT & No & 8 & Pichilemu (VI) \\
\hline 4 & $\mathrm{~F}$ & 40 & $50 \%$ & $2^{\circ}$ & Fuego & Sí & IOT & No & 5 & La Florida (RM) \\
\hline 5 & $M$ & 44 & $53 \%$ & $2^{\circ}$ & Fuego & Sí & IOT & No & 4 & Valparaíso (V) \\
\hline 6 & $\mathrm{~F}$ & 37 & $14 \%$ & $3^{\circ}$ & Fuego & Sí & IOT & Sí & 5 & Temuco (IX) \\
\hline 7 & $M$ & 35 & $35 \%$ & $3^{\circ}$ & Fuego & Sí & IOT & Sí & 6 & $\begin{array}{l}\text { Puente Alto } \\
\text { (RM) }\end{array}$ \\
\hline 8 & $M$ & 31 & $23 \%$ & $2^{\circ}$ & Fuego & Sí & IOT & No & 8 & San Bdo. (RM) \\
\hline 9 & $M$ & 34 & $58 \%$ & $2^{\circ}$ & Fuego & Sí & IOT & No & 8 & Zapallar (V) \\
\hline 10 & $M$ & 29 & $29 \%$ & $2^{\circ}$ & Fuego & Sí & IOT & No & 7 & Pto. Montt (X) \\
\hline
\end{tabular}

I.I: injuria inhalatoria; IOT: intubación orotraqueal; TQT: traqueostomía; IDE: injerto dermoepidérmico; $\mathrm{N}^{\circ}$ MOF: cantidad de sesiones de motricidad orofacial. / M: Masculino; F: Femenino; F+E: fuego + explosión.

Nota: profundidad e IDE sólo relacionado con quemadura orofacial. No considera otras zonas corporales que podrían haber requerido injertos y/o presentar diferencias en relación a su profundidad.

El $80 \%$ de los casos correspondió a hombres, con una media de edad de 38,2 años (rango [28-55], D.S: $\pm 8,85) .40 \%$ de los pacientes provenía de la región Metropolitana, $20 \%$ de la región de Valparaíso y $40 \%$ de otras regiones del país. La media de SCQ fue de un $35,8 \%$ (rango [9\%-58\%]; D.S: $\pm 17,08$ ), donde $80 \%$ de los casos presentó quemaduras de $2^{\circ}$ grado y sólo $20 \%$ quemaduras de $3^{\text {er }}$ grado. $30 \%$ del grupo requirió injerto dermoepidérmico (IDE) en región facial. El mecanismo más frecuente fue fuego directo en $70 \%$ de los casos. Por otra parte, la totalidad de los pacientes presentó injuria inhalatoria concomitante y requirió soporte ventilatorio por medio de intubación orotraqueal -IOT- o traqueostomía -TQT-. LOS pacientes recibieron una mediana de 6 sesiones (rango [4-8], D.S: $\pm 1,59$ ) fonoaudiológicas de motricidad orofacial. 
Tabla 2

Caracterización del tiempo transcurrido hasta la primera evaluación Fonoaudiológica.

\begin{tabular}{lcc}
\hline & $\begin{array}{c}\text { Días transcurridos desde quemadura hasta } 1^{\circ} \\
\text { evaluación fonoaudiológica (FNA) }\end{array}$ & $\begin{array}{c}\text { Días transcurridos desde ingreso hospitalario hasta } 1^{\circ} \\
\text { evaluación fonoaudiológica (FNA) }\end{array}$ \\
\hline Mediana & 23,5 & 17,5 \\
Media & 26,8 & 22,8 \\
D.S & 15,19 & 15,08 \\
Rango & $11-61$ & $6-55$ \\
\hline
\end{tabular}

Tal como puede ser observado en la tabla 2, el tiempo transcurrido hasta la primera evaluación fonoaudiológica fue variado, realizándose con una mediana de 23,5 días posterior a la injuria (rango [1161], D.S: $\pm 15,19$ ) y 17,5 días (rango [6-55], D.S: \pm $15,08)$ desde el ingreso hospitalario.

De acuerdo a los hallazgos de la evaluación miofuncional inicial (tabla 3) y comparando con los valores normativos descritos con anterioridad, $100 \%$ de los pacientes presentó disminución en los movimientos de apertura bucal vertical (media de $23,1 \mathrm{~mm}$; rango [18-27] $\mathrm{mm}$ y $\mathrm{D} . \mathrm{S} \pm 2,9$ ) y horizontal (media: 36,1 mm; rango [31-41] y D.S $\pm 2,9$ ), además de disminución en los movimientos de mímica facial (media: 15,7 puntos, rango [6-26] y D.S $\pm 7,0$ ).

Tabla 3

Comparativo en los valores de apertura bucal y mímica facial en evaluación inicial y posterior a la intervención fonoaudiológica.

\begin{tabular}{|c|c|c|c|c|c|c|c|c|c|}
\hline \multirow[b]{2}{*}{ Caso } & \multicolumn{3}{|c|}{ A.B Vertical (mm) } & \multicolumn{3}{|c|}{ A.B Horizontal $(\mathrm{mm})$} & \multicolumn{3}{|c|}{ Mímica Facial (Puntos) } \\
\hline & Inicio & Final & $\Delta$ & Inicio & Final & $\Delta$ & Inicio & Final & $\Delta$ \\
\hline 1 & 18 & 25 & 7 & 31 & 36 & 5 & $6 / 40$ & $26 / 40$ & 20 \\
\hline 2 & 21 & 23 & 2 & 35 & 39 & 4 & $17 / 40$ & $32 / 40$ & 15 \\
\hline 3 & 25 & 33 & 8 & 36 & 45 & 9 & $14 / 40$ & $34 / 40$ & 20 \\
\hline 4 & 26 & 32 & 6 & 34 & 42 & 8 & $26 / 40$ & $32 / 40$ & 6 \\
\hline 5 & 22 & 30 & 8 & 41 & 45 & 4 & $24 / 40$ & $34 / 40$ & 10 \\
\hline 6 & 20 & 30 & 10 & 35 & 42 & 7 & $11 / 40$ & $23 / 40$ & 12 \\
\hline 7 & 23 & 31 & 8 & 37 & 45 & 8 & $7 / 40$ & $22 / 40$ & 15 \\
\hline 8 & 26 & 30 & 4 & 34 & 44 & 10 & $24 / 40$ & $36 / 40$ & 20 \\
\hline 9 & 27 & 35 & 8 & 40 & 47 & 7 & $14 / 40$ & $32 / 40$ & 18 \\
\hline \multirow[t]{2}{*}{10} & 23 & 31 & 8 & 38 & 46 & 8 & $14 / 40$ & $28 / 40$ & 14 \\
\hline & & & $p$ & & & $p$ & & & $p$ \\
\hline Mediana & 23 & 30,5 & & 35,5 & 44,5 & & 14 & 32 & \\
\hline Media & 23,1 & 30 & $.005^{*}$ & 36,1 & 43 & $.005^{*}$ & 15,7 & 29,9 & $0.005^{*}$ \\
\hline D.S & $\pm 2,9$ & $\pm 3,5$ & & $\pm 2,9$ & $\pm 3,4$ & & $\pm 7,0$ & $\pm 4,8$ & \\
\hline
\end{tabular}

A.B: apertura bucal; $\Delta$ : diferencia $* p<0,05$ 
Realizando el análisis comparativo pre y post terapia fonoaudiológica, tal como se puede observar en la tabla 3, existen diferencias estadísticamente significativas $(Z=-2,8 ; p=0,005)$ en la variación numérica de las tres variables: apertura bucal vertical, horizontal y mímica facial para el grupo en estudio.
Adicionalmente, al realizar el análisis cuantitativo caso a caso, se evidenciaron mejoras en los valores de cada parámetro en todos los casos; con incremento de [2-10] mm en la apertura bucal vertical, [4-10] $\mathrm{mm}$ en la apertura bucal horizontal y [6-20] puntos en el Clinical Score for Facial MimicProtocol.

Tabla 4

Nivel de auto percepción de retracción pre y post terapia fonoaudiológica.

\begin{tabular}{|c|c|c|c|c|}
\hline \multirow[b]{2}{*}{ Caso } & \multirow[b]{2}{*}{ Retracción } & \multicolumn{3}{|c|}{ Valoración } \\
\hline & & Pre & Post & $\Delta$ \\
\hline 1 & Sí & 2 & 5 & 3 \\
\hline 2 & Sí & 4 & 6 & 2 \\
\hline 3 & Sí & 3 & 6 & 3 \\
\hline 4 & Sí & 3 & 6 & 3 \\
\hline 5 & Sí & 3 & 5 & 2 \\
\hline 6 & Sí & 4 & 6 & 2 \\
\hline 7 & Sí & 2 & 5 & 3 \\
\hline 8 & Sí & 2 & 4 & 2 \\
\hline 9 & Sí & 3 & 7 & 4 \\
\hline \multirow[t]{5}{*}{10} & Sí & 3 & 6 & 3 \\
\hline & & & & $p$ \\
\hline & Mediana & 3.0 & 6,0 & \\
\hline & Media & 2.9 & 5.6 & $.004^{*}$ \\
\hline & D.S. & $\pm 0,74$ & $\pm 0,84$ & \\
\hline
\end{tabular}

${ }^{*} p<0,05$

Nota: Valoración en escala de 1 a 7, donde 1 constituye sensación de máxima retracción y 7 sensación de mínima retracción.

Por otra parte, el nivel de auto percepción de los pacientes respecto al estado de retracción (tabla 4) mejoró posterior a la terapia fonoaudiológica, donde la totalidad del grupo percibió y autovaloró este ítem en forma positiva, con diferencias estadísticamente significativas $(z=-2,86 ; p=0,004)$. 
Tabla 5

Cantidad de sesiones recibidas por paciente al momento de realizar la última medición, estado de la terapia, conducta y situación al alta.

\begin{tabular}{|c|c|c|c|c|}
\hline Caso & $\begin{array}{c}\text { Cantidad de sesiones } \\
\text { MOF } \\
\end{array}$ & Estado Terapia & Situación al alta & $\begin{array}{c}\text { Conducta de } \\
\text { Rebabilitación }\end{array}$ \\
\hline 1 & 6 & I & A.H & D.H.B \\
\hline 2 & 4 & 1 & A.H & D.H.B \\
\hline 3 & 8 & $\mathrm{~F}$ & A.H & D.H.B \\
\hline 4 & 5 & I & A.H & D.H.B \\
\hline 5 & 4 & I & T.H.B & $*$ \\
\hline 6 & 5 & I & T.H.B & $*$ \\
\hline 7 & 6 & I & A.H & D.H.B \\
\hline 8 & 8 & I & T.H.B & $*$ \\
\hline 9 & 8 & $\mathrm{~F}$ & T.H.B & $*$ \\
\hline 10 & 7 & I & T.H.B & $*$ \\
\hline
\end{tabular}

I: Inconcluso; F: Finalizado; A.H: Alta Hospitalaria; D.H.B: Derivación a Fonoaudiología en Hospital Base; T.H.B: Traslado Hospital Base; *: Conducta a definir en Hospital Base

Finalmente, y de acuerdo con lo observado en la tabla 5, es importante destacar que sólo $20 \%$ de los pacientes logró concluir su intervención cumpliendo los objetivos terapéuticos y con mejoras significativas en los parámetros evaluados, para lo cual requirieron un total de 8 sesiones. El $80 \%$ egresó del hospital con tratamiento fonoaudiológico inconcluso, es decir, no cumpliendo los objetivos propuestos al inicio de la intervención (ver apartado metodología). En este último grupo, al egreso de nuestra institución, 50\% fue trasladado a su hospital de origen, mientras que $50 \%$ restante fue dado de alta con controles en su hospital de referencia.

\section{Discusión}

El presente estudio tuvo como objetivo describir y evidenciar los efectos de la terapia fonoaudiológica miofuncional en un grupo de personas con quemaduras orofaciales de $2^{\circ}$ y $3^{\text {er }}$ grado, mostrando una serie de diez casos con desorden miofuncional orofacial secundario a la quemadura.

De acuerdo con los resultados, fue posible evidenciar que la totalidad de los sujetos evaluados presentaron algún grado de limitación en la apertura bucal y movimientos faciales. Estos hallazgos concuerdan con lo expuesto en las investigaciones de Clayton et al., 2015a, 2015b; Clayton, Ward, \& Maitz, 2017; Magnani et al., 2015; Ramos et al., 2009. Las alteraciones de motricidad orofacial en personas quemadas dependerán principalmente del agente y 
profundidad de la quemadura, siendo frecuente encontrar limitación de la apertura bucal (pudiendo llegar a la microstomía en casos severos), movimientos mandibulares y masticatorios, movimientos de la mímica facial, incluso movimientos cervicales (Magnani et al., 2015). En este sentido, aquellos pacientes con quemadura de $2^{\circ}$ y $3^{\text {er }}$ grado constituyen grupos de riesgo para desarrollar alteraciones en las funciones del sistema estomatognático y movimientos de mímica facial (Magnani et al., 2015).

Por su parte la terapia fonoaudiológica miofuncional, expuesta en el presente artículo, incrementó de forma significativa las medidas de apertura bucal (vertical y horizontal) y movilidad de la mímica facial, además de disminuir la autopercepción de retracción en la totalidad de los casos al finalizar las sesiones terapéuticas. Este último punto es destacable, pues a diferencia de las investigaciones previa, que no consideraban la autopercepción del paciente, la actual investigación indicaría que la terapia fonoaudiológica miofuncional además, beneficia la percepción del paciente en relación al estado de retracción del tejido, pudiendo producir mejoras en la sensación de bienestar del paciente, y por consecuente, en su calidad de vida.

Los hallazgos de la presente investigación apoyan la evidencia ya disponible, y son concordantes con los resultados de las intervenciones expuestas por Clayton et al., 2017, 2015a, 2015b; Ramos et al., 2009; Toledo, 2003, donde se ha manifestado la efectividad de la intervención fonoaudiológica miofuncional en esta población.

De las secuelas en quemaduras orofaciales, gran relevancia tiene la microstomía o disminución de la apertura bucal, pues ocasiona secuelas funcionales que pueden repercutir en la comunicación verbal (principalmente articulación), alimentación oral (contención oral de alimentos o líquidos al comer), higiene bucal o incluso, ocasionar complicaciones futuras asociadas por ejemplo, al realizar tratamientos dentales. En casos severos de microstomía, esta deberá ser corregida por medio de cirugía reparadora (Zweifel et al., 2010). En este sentido, es útil una intervención precoz con técnicas de rehabilitación oral que permitan prevenir casos severos, asegurar funcionalidad y evitar cirugías reparadoras.

Si bien se han descrito y evidenciado otras técnicas en la prevención de la microstomía (Borges et al., 2011; Vana, Fontana, Reis, \& Ferreira, 2009), como por ejemplo el uso de órtesis bucales, en el presente artículo no fueron consideradas pues podrían interferir en los resultados obtenidos y limitar la evidencia de la intervención fonoaudiológica miofuncional. Sin embargo, es relevante destacar que el adecuado uso de órtesis (como expansores o máscaras faciales) sumado a la terapia miofuncional podría generar mejores resultados a nivel funcional y estético, entendiendo que estos procedimientos son complementarios y no excluyentes.

El manejo de secuelas en quemaduras orofaciales aún constituye una zona gris en diversas profesiones, donde existe complemento en el trabajo realizado entre Fonoaudiólogos, Kinesiólogos y Terapeutas Ocupacionales (Clayton et al., 2017; Clayton, Whitney, O'Loughlin, Crouch, \& Patterson, 2007). De esta forma es fundamental el trabajo mancomunado y realizado por las otras disciplinas, cada una con objetivos orientados a su área de trabajo, pero que 
confluyen en el bienestar de la persona. Si bien, la presente investigación sólo destacó los beneficios de la terapia fonoaudiológica miofuncional, muy importante es la comunicación y los objetivos en común con el equipo de Terapia Ocupacional, quiénes podrán contribuir con la implementación de órtesis bucales para mejorar los resultados en la intervención de la microstomía o manejo de cicatrices; o con el equipo de Kinesiología, quiénes pueden ser de apoyo en relación a la movilización y/o compresión según sea el caso.

Al momento de trabajar con quemaduras faciales, es importante que el terapeuta presente algunas consideraciones y maneje conocimientos respecto a procesos fisiopatológicos frecuentes como cicatrización (normal y patológica) e injertos.

El proceso de cicatrización involucra una secuencia de eventos en un tiempo determinado, pudiéndose ver interferidos por factores locales y sistémicos. El tejido cicatrizal ocasiona cambios en la piel, determinando características propias que lo diferencian de la piel adyacente en cuanto a color, grosor, elasticidad, textura y/o grado de contracción (Glat \& Longaker, 1997). Las cicatrices son frecuentes en aquellos pacientes con quemaduras intermedias y/o profundas ( $2^{\circ}$ y $3^{\text {er }}$ grado respectivamente), donde muchas veces predominan las cicatrices hipertróficas. Estas se caracterizan por una respuesta desorganizada que altera la adecuada síntesis de colágeno; al ser esta producción irregular, se precipita la aparición de una cicatriz por la formación de tejido de granulación. En este sentido, técnicas como las implementadas en el presente estudio (ejercicios orofaciales, manipulación pasiva o masoterapia), contribuyen en la mantención o recuperación -según sea el caso- del rango de movimiento orofacial y la flexibilización del tejido cicatrizal.

En el caso de los injertos, es crucial la comunicación con el equipo de cirugía plástica, quiénes autorizarán la manipulación de la zona injertada para favorecer la movilidad de la misma. De acuerdo al modelo de intervención interdisciplinario que existe en el HUAP, siempre fue consultado con el equipo quirúrgico tratante antes de iniciar la intervención; de esta forma los tres casos que requirieron injertos comenzaron una rehabilitación orofacial temprana (incluso antes de la realización de los injertos), retomando las sesiones una vez que el equipo de cirugía plástica autorizara.

Pese a los resultados positivos en la intervención realizada, el presente estudio no se encuentra exento de limitaciones.

La intervención fonoaudiológica fue realizada en fase hospitalaria, lo cual constituye una limitación en relación a la posibilidad de realizar la totalidad de los procedimientos evaluativos y terapéuticos, además del adecuado seguimiento. Por lo general, la intervención de la motricidad orofacial debiera ser iniciada en fase hospitalaria y continuar en forma ambulatoria (Toledo, 2003), para el monitoreo continuo del proceso cicatrizal, el cual continúa hasta 2 años posterior a la quemadura y también para monitorear las posibles secuelas en funciones orofaciales y mímica facial. Durante la fase hospitalaria, a diferencia de la fase ambulatoria, el paciente aún se encuentra siendo sometido a diversos procedimientos médicos-quirúrgicos (algunos de ellos de carácter invasivo), además de ver interferido su rendimiento por el uso fármacos o dolor, lo que dificultará su colaboración y adherencia con la 
terapia. Sumado a lo anterior y pese a que no ha sido descrito en el presente artículo, los pacientes quemados del HUAP presentan alta comorbilidad con trastornos de origen psiquiátrico, lo cual dificulta su manejo. Estas condiciones clínicas podrían ser motivos que explicarían el por qué no todos los pacientes pudieran recibir las dos sesiones semanales; o que la no todos adhirieran a la terapia indirecta, lo cual dificultó que los pacientes complementaran el trabajo realizado en las sesiones.

Sumado a lo ya expuesto, también existen limitaciones desde el punto de vista metodológico con los instrumentos de evaluación. En primer lugar, la pauta de exploración de la motricidad orofacial utilizada en el estudio fue diseñada por el autor, es de carácter cualitativo y subjetivo, y no se encuentra validada ni publicada en el medio; todo lo anterior resta validez y confiabilidad. En segundo lugar, para evaluar funcionalidad de la mímica facial se debió utilizar un protocolo creado inicialmente para pacientes con parálisis facial periférica. Sin embargo, considerando la similitud en el comportamiento clínico de ambos grupos, esta herramienta ha sido utilizada con pacientes quemados previamente (Magnani et al., 2015). A la fecha, aún no existen protocolos debidamente validados que evalúen dichos aspectos en esta población, lo cual restringe la posibilidad de valorar la funcionalidad de la mímica facial y tener una referencia comparativa dentro del limitado grupo que constituyen los pacientes quemados.

Otra limitación desde el punto de vista metodológico, lo constituyen la ausencia de datos vinculados al inicio de las sesiones de motricidad orofacial, como por ejemplo, cantidad de días desde la quemadura hasta el inicio de la primera sesión de MOF, o desde la evaluación fonoaudiológica hasta el inicio de la primera sesión de MOF; que permitirían aportar mayor solidez a la efectividad de la intervención realizada. Si bien, se aportan datos en relación a la primera atención fonoaudiológica, estos hacen referencia al momento de la evaluación inicial y no necesariamente corresponde a la intervención específica de lo mencionado en el presente artículo. De esta forma sería recomendable, considerar este factor en estudios posteriores al momento de analizar los resultados de la intervención.

Al ser el HUAP centro de referencia nacional y de atención exclusiva hospitalaria, muchas veces los pacientes no concluyen su tratamiento y/o son derivados para continuar su rehabilitación en otros lugares al momento del alta (sin la certeza de que realmente la reciban) tal como pudo ser evidenciado en la tabla 5. Por otra parte, la misma geografía de Chile dificulta el acceso y las oportunidades que pueden presentar los pacientes provenientes de regiones extremas. En este sentido, fue posible evidenciar que la cantidad de sesiones fonoaudiológicas no fue uniforme en la totalidad del grupo, ya que de acuerdo a la procedencia y exigencias del servicio algunos pacientes debieron ser trasladados a su hospital de origen o continuar la rehabilitación en otros centros, sin saber si en dichos lugares contarían con la totalidad de profesionales necesarios para dar respuesta a los requerimientos del paciente. En aquellos casos, medidas como educación al paciente y/o familiar (en casos que existían redes de apoyo) debieron ser implementadas para asegurar la efectividad y continuidad parcial de la intervención; además de la creación de pautas y 
programas de ejercicios al momento del alta hospitalaria. Este último punto no fue posible de realizar en la totalidad de los casos egresados de nuestro servicio, fundamentado principalmente en el egreso repentino y la demanda del sistema ante requerimientos de camas para nuevos ingresos de pacientes graves y de alta complejidad.

Por otra parte, las políticas públicas actuales no consideran la intervención fonoaudiológica como parte de los beneficios a los que debiera acceder el paciente quemado. Sumado a lo anterior, el nivel de desconocimiento por parte del equipo o de los mismos fonoaudiólogos, además de la escasa evidencia, capacitación o formación en esta temática limitan la inclusión de la profesión en el abordaje del paciente quemado. De acuerdo a lo planteado en párrafos anteriores, y teniendo en cuenta que el proceso de cicatrización se extiende por aproximadamente 2 años, es fundamental realizar sesiones de seguimiento con el objetivo de mantener los resultados obtenidos en fase hospitalaria, mejorar aquellos déficits que aún persisten o pesquisar secuelas que podrían aparecer a posterior. Por lo tanto, pese a lo fundamental que podría ser el seguimiento y la continuidad de la atención del proceso de rehabilitación de un paciente quemado, las posibilidades reales de seguimiento y continuidad de la terapia fonoaudiológica, aún en otro centro, es baja y limitada.

Pese a las limitaciones antes mencionadas y a lo acotado del grupo de estudio, el presente estudio corresponde a la primera serie de casos relacionado con la intervención de la motricidad orofacial en pacientes quemados realizado en nuestro país. Los aportes de la fonoaudiología en esta área son recientes, siendo fundamental contribuir con la evidencia que permita validar la disciplina en el abordaje del paciente quemado y en un futuro ser considerados en los programas de políticas públicas vinculados con la atención de estos pacientes. Junto a ello la formación de profesionales fonoaudiólogos que permitan satisfacer las demandas y necesidades de este particular grupo, tanto en el área de motricidad orofacial como en otras áreas de atención fonoaudiológica.

Futuras investigaciones deben ser realizadas en esta población, no sólo en Chile, sino en el resto del mundo pues las características clínicas varían según la población y complejidad de cada centro. Sería interesante contar con cuestionarios de calidad de vida vinculadas a las secuelas de índole fonoaudiológica, además de investigaciones enfocadas en las diversas áreas de atención fonoaudiológica (voz, motricidad orofacial y deglución), con el objetivo de contribuir con la evidencia científica de la disciplina y objetivar los aportes de la fonoaudiología en esta nueva área de intervención.

\section{Síntesis final}

La incorporación de la fonoaudiología en forma activa en los equipos de quemados cada vez cobra mayor fuerza. Esta nueva área de intervención para la disciplina permite un abordaje integral y precoz desde la fase hospitalaria que permitirá disminuir las secuelas posteriores.

El presente artículo pone en evidencia los aportes de la fonoaudiología desde el ámbito de la motricidad orofacial, enfatizando acerca de los beneficios de la 
intervención fonoaudiológica temprana en un grupo de pacientes quemados.

De este modo, es fundamental una intervención oportuna que permita disminuir las secuelas a las que se encuentran expuestos los pacientes quemados, así como la continuidad del tratamiento para realizar un adecuado monitoreo del proceso. Por otra parte, se vuelve prioritaria la formación de colegas capacitados en cubrir las necesidades de estos pacientes y contribuir con la evidencia del trabajo fonoaudiológico en esta población; de manera tal, que a futuro nuestra disciplina sea considerada en los programas de políticas públicas dirigidas a estos pacientes.

\section{Referencias}

Albornoz, C., Villegas, J., Peña, V., \& Whittle, S. (2013). Epidemiología del paciente gran quemado adulto en Chile: experiencia del Servicio de Quemados del Hospital de la Asistencia Pública de Santiago. Revista médica de Chile, 141(2), 181-186. https://doi.org/10.4067/S003498872013000200006

Borges, G., Vieira, A. C., \& Barreto, M. (2011). Queimadura de face: abordagem fonoaudiológica na prevenção de microstomia. Revista Brasileira de Queimaduras, 10(1), 3538.

Brooks, J., \& Hammond, J. S. (1986). Nonverbal communication: role of the speech pathologist on the burn team. The Journal of Burn Care \& Rehabilitation, 7(1), 42-44.

Clayton, N, Whitney, S., O'Loughlin, G., Crouch, A., \& Patterson, M. (2007). Speech Pathologist. En D. Edgar \& A. Katsu (Eds.), Burn Survivor Rehabilitation: Principles and guidelines for the allied health professional (pp. 135-157). Brisbrane: Australian and New Zealand Burns Association. Recuperado de http://research.kaums.ac.ir/UploadedFiles/trauma\%20\%20nursing/Burn\%20relabilitation.PDF
Clayton, N., Ellul, G., Ward, E., Scott, A., \& Maitz, P. (2017). Orofacial Contracture Management: Current Patterns of Clinical Practice in Australian and New Zealand Adult Burn Units. Journal of Burn Care \& Research: Official Publication of the American Burn Association, 38(1), e204-e211. https://doi.org/10.1097/BCR.0000000000000351

Clayton, N., Ward, E., \& Maitz, P. (2015a). Full thickness facial burns: Outcomes following orofacial rehabilitation. Burns: Journal of the International Society for Burn Injuries, 41(7), 1599-1606.

https://doi.org/10.1016/j.burns.2015.04.003

Clayton, N., Ward, E., \& Maitz, P. (2015b). Orofacial contracture management outcomes following partial thickness facial burns. Burns: Journal of the International Society for Burn Injuries, 41(6), 1291-1297. https://doi.org/10.1016/j.burns.2015.02.015

Clayton, N., Ward, E., \& Maitz, P. (2017). Intensive swallowing and orofacial contracture rehabilitation after severe burn: A pilot study and literature review. Burns: Journal of the International Society for Burn Injuries, 43(1), e7-e17. https://doi.org/10.1016/j.burns.2016.07.006

Glat, P., \& Longaker, M. (1997). Wound Healing. En C. Thorne, S. Aston, \& R. Beasly (Eds.), Grabb And Smith's Plastic Surgery (Edición: 7th revised edition, pp. 3-12). Philadelphia: Lippincott Williams And Wilkins.

Hop, M. J., Langenberg, L. C., Hiddingh, J., Stekelenburg, C. M., van der Wal, M. B. A., Hoogewerf, C. J., ... Middelkoop, E. (2014). Reconstructive surgery after burns: a 10-year follow-up study. Burns: Journal of the International Society for Burn Injuries, 40(8), 1544-1551. https://doi.org/10.1016/j.burns.2014.04.014

ISBI Practice Guidelines Committee, Steering Subcommittee, \& Advisory Subcommittee. (2016). ISBI Practice Guidelines for Burn Care. Burns: Journal of the International Society for Burn Injuries, 42(5), 953-1021. https://doi.org/10.1016/j.burns.2016.05.013

Magnani, D. M., Sassi, F. C., Vana, L. P. M., Alonso, N., \& Andrade, C. R. F. de. (2015). Evaluation of oral-motor movements and facial mimic in patients with head and neck burns by a public service in Brazil. Clinics (Sao Paulo, Brazil), 70(5), 339-345. https://doi.org/10.6061/clinics/2015(05)06

Mezitis, M., Rallis, G., \& Zachariades, N. (1989). The normal range of mouth opening. Journal of Oral and Maxillofacial Surgery: Official Journal of the American Association of Oral and Maxillofacial Surgeons, 47(10), 1028-1029. 
Ministerio de Salud de Chile. (2016). Guía Clínica: Manejo del paciente gran quemado. Recuperado de http://www.bibliotecaminsal.cl/wp/wpcontent/uploads/2016/04/GPC-GRAN-QUEMADO-FINAL18-MARZO-2016_DIAGRAMADA.pdf

Pavez, A., \& Tobar, R. (2016). Intervención fonoaudiológica en quemados: relato de un caso en el Hospital de Urgencia Asistencia Pública, Chile. Revista Brasileira de Queimaduras, 15(4), 278-282.

Ramos, E., Danda, F., Araujo, F., Regis, R., \& Silva, H. (2009). Tratamento fonoaudiológico em queimadura orofacial. Revista Brasileira de Queimaduras, 8(2), 70-74.

Rumbach, A. F., Clayton, N. A., Muller, M. J., \& Maitz, P. K. M. (2016). The speech-language pathologist's role in multidisciplinary burn care: An international perspective. Burns: Journal of the International Society for Burn Injuries, 42(4), 863-871.

https://doi.org/10.1016/j.burns.2016.01.011

Salles, A. G., Toledo, P. N., \& Ferreira, M. C. (2009). Botulinum toxin injection in long-standing facial paralysis patients: improvement of facial symmetry observed up to 6 months. Aesthetic Plastic Surgery, 33(4), 582-590. https://doi.org/10.1007/s00266-009-9337-9

Snyder, C., \& Ubben, P. (2003). Use of speech pathology services in the burn unit. The Journal of Burn Care \& Rehabilitation, 24(4), 217-222. https://doi.org/10.1097/01.BCR.0000075969.19229.EE

Stoddard, F. J., Ryan, C. M., \& Schneider, J. C. (2014). Physical and psychiatric recovery from burns. The Surgical Clinics of North America, 94(4), 863-878. https://doi.org/10.1016/j.suc.2014.05.007

Toledo, P. (2003). Conhecimentos essenciais parar atender bem os pacientes queimados. São José dos Campos: Pulso.

Vana, L. P., Fontana, C., Reis, J. O. G. dos, \& Ferreira, M. (2009). Tratamento de microstomia grave: relato de caso. Revista Brasileira de Queimaduras, 8(2), 75-78.

Wei, Y., \& Li-Tsang, C. W. (2017). Rehabilitation of Patients with Facial Burn Injury: Principles and Practice Experiences. JSM Burns and Trauma, 2(3), 1-5.

Williams, A. I., \& Baker, B. M. (1992). Advances in burn care management: role of the speech-language pathologist. The Journal of Burn Care \& Rehabilitation, 13(6), 642-649.

Zweifel, C. J., Guggenheim, M., Jandali, A. R., Altintas, M. A., Künzi, W., \& Giovanoli, P. (2010). Management of microstomia in adult burn patients revisited. Journal of Plastic, Reconstructive \& Aesthetic Surgery: JPRAS, 63(4), e351-357. https://doi.org/10.1016/j.bjps.2009.10.026 